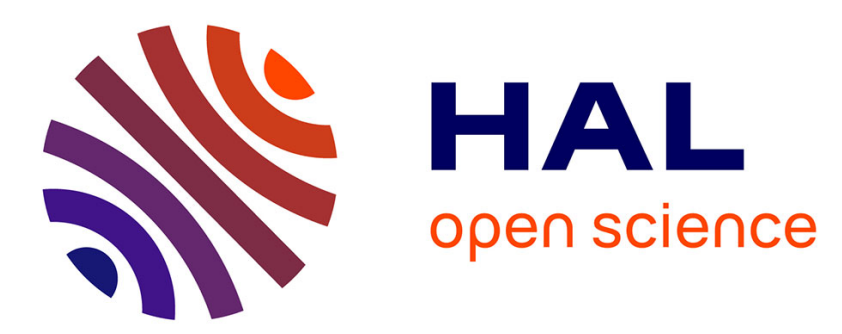

\title{
Plasma holograms for ultrahigh-intensity optics
}

\author{
A. Leblanc, A. Denoeud, L. Chopineau, G. Mennerat, P. Martin, F. Quéré
}

\section{To cite this version:}

A. Leblanc, A. Denoeud, L. Chopineau, G. Mennerat, P. Martin, et al.. Plasma holograms for ultrahigh-intensity optics. Nature Physics, 2017, 13 (5), pp.440-443. 10.1038/NPHYS4007 . hal02276885

\section{HAL Id: hal-02276885 \\ https://hal.science/hal-02276885}

Submitted on 4 Sep 2019

HAL is a multi-disciplinary open access archive for the deposit and dissemination of scientific research documents, whether they are published or not. The documents may come from teaching and research institutions in France or abroad, or from public or private research centers.
L'archive ouverte pluridisciplinaire HAL, est destinée au dépôt et à la diffusion de documents scientifiques de niveau recherche, publiés ou non, émanant des établissements d'enseignement et de recherche français ou étrangers, des laboratoires publics ou privés. 


\title{
Plasma holograms for ultrahigh-intensity optics
}

\author{
A. Leblanc, A. Denoeud, L. Chopineau, G. Mennerat, Ph. Martin and F. Quéré^
}

The manipulation of ultraintense laser beams gets increasingly challenging with growing laser peak power, as the breakdown of conventional optics imposes ever larger beam diameters. Using compact plasma-based optical elements to control or even generate such beams ${ }^{1-4}$ is a promising approach, since plasmas can sustain considerable light intensities. We introduce a new type of plasma optics, called plasma holograms, by initiating plasma expansion on a flat solid target with a holographic prepulse beam focus. A modulated plasma surface then grows out of the target after ionization, which can be used for several picoseconds to diffract and spatially shape ultraintense laser beams. On the basis of this concept, we demonstrate the generation of fork plasma gratings, which we use to induce optical vortices on a femtosecond laser beam as well as its high-order harmonics, at intensities exceeding $10^{19} \mathrm{~W} \mathrm{~cm}^{-2}$. These plasma holograms open up a whole new range of possibilities for the manipulation of ultraintense lasers and the generation of structured coherent short-wavelength sources.

At present, plasma mirrors are the most widely used plasmabased optical elements. These are dense plasmas produced at the surface of solid targets ionized by intense femtosecond laser pulses ${ }^{5}$. Due to their solid-like density $\left(n_{\mathrm{e}} \approx 10^{23} \mathrm{~cm}^{-3}\right)$ and to their limited expansion into the vacuum on a picosecond timescale, such plasmas specularly reflect these pulses, just like ordinary mirrors do for lowintensity laser beams. They have already demonstrated a variety of advanced applications as single-shot, compact, and cheap optical devices, from contrast improvement ${ }^{6,7}$, frequency-doubling ${ }^{8}$ and tight focusing of ultraintense beams ${ }^{9}$, to the generation of highorder harmonics ${ }^{1,10,11}$ and isolated attosecond pulses ${ }^{12}$.

More recently, a new type of plasma optics closely related to plasma mirrors has been demonstrated, called transient plasma gratings $^{13}$. In this case, the local fluence of the laser beam that creates the plasma is sinusoidally modulated along the surface of a flat solid target. This results in a spatial variation of the expansion velocity of the plasma into the vacuum ${ }^{14}$, thus leading to the growth in time of a modulated plasma surface. Such structures act as phase gratings on any subsequent laser beam, and can sustain ultrahigh laser intensities ${ }^{13}$. Their time-resolved surface profile has been measured by in situ ptychography ${ }^{15}$.

The starting point of the present work is that plasma expansion can be initiated by arbitrarily complex interference patterns: one can thus trigger the growth of sophisticated structures at the surface of dense plasmas by crossing two or more synchronized 'prepulse' beams on target, and then diffract a main beam from this structure. This simple idea considerably extends the range of possibilities in the manipulation of intense lasers with plasmas.

The initial interference pattern can in particular be obtained by using holographic techniques (Fig. 1a). One prepulse can, for instance, consist of a reference beam with a flat spatial phase, while the other constitutes the 'object beam' with a more complex spatial phase profile. Since the prepulse beams can be small $(\leq 1 \mathrm{~cm})$ and of moderate energy (a few millijoules), controlling their phase profile is straightforward, for example, by inserting an 'object' in the beam prior to focusing on target, or even using programmable beam shaping devices such as spatial light modulators. As in standard holography, when a subsequent ultraintense main beam is diffracted off the plasma structure initiated by this holographic pattern (Fig. 1b), the diffracted orders are replicas of the prepulse 'object beam' (Fig. 1c), now of potentially much higher energy. This scheme thus produces transient phase holograms made of plasmas, suitable for manipulating ultraintense laser beams.

We demonstrate this idea experimentally by creating fork plasma gratings. Fork gratings are the holographic patterns obtained by interfering a reference beam with a beam carrying an optical vortex, such as a Laguerre-Gaussian beam of azimuthal index $|l| \geq 1$ (refs 16,17). Their periodicity progressively varies along the grating lines, until $|l|$ lines disappear as one passes across the optical vortex (with $l$ being the vortex topological charge $)^{17}$. Inversely, vortices are routinely generated on light or even particle beams by diffraction off amplitude or phase fork gratings. The $k$ th diffracted order then has helical phase fronts, associated with a vortex of topological charge $k \times l$ (ref. 17).

This experiment was carried out on UHI100, the $100 \mathrm{TW}-25 \mathrm{fs}$ $800 \mathrm{~nm}$ laser at CEA-IRAMIS. Starting from this single laser beam, a simple optical set-up (see Supplementary Information) provides two independent prepulse beams and one main ultraintense beam, all with controlled relative delays. When synchronized, the two prepulses create a sinusoidal interference pattern on target of line spacing $d=3.75 \mu \mathrm{m}$, shown in Fig. 2a. This pattern is turned into a fork by inducing an optical vortex on one prepulse beam using a $2 \pi$ spiral phase plate (Fig. $1 \mathrm{a})^{17}$, while the other prepulse beam remains unchanged. After an adjustable delay, the main ultraintense laser beam can be diffracted off the resulting plasma structure. This can be achieved with the prepulse interference fringes either perpendicular (classical diffraction geometry, illustrated in Fig. 1) or parallel (conical diffraction geometry) to the main beam incidence plane, simply by changing the positions of the two prepulse beams (see Supplementary Information). Both configurations have been tested in the present work. To clearly separate the different diffraction orders, we diaphragmed this main laser beam to $12.5 \mathrm{~mm}$, leading to a focal spot of $20 \mu \mathrm{m}$ diameter $(\approx 5-6$ lines of the plasma grating) and a peak intensity of $\approx 10^{17} \mathrm{~W} \mathrm{~cm}^{-2}$ on target.

The fork interference pattern is expected to trigger the growth of a fork plasma grating. Reflecting the ultraintense main beam off this structure should then lead to diffracted beams carrying optical vortices. A signature of such beams, which comes from their phase singularities, is the cancellation of the light intensity around the vortex axis. This typically leads to beams with a ring structure, as is the case for Laguerre-Gaussian beams ${ }^{17}$. This is precisely what we observe experimentally: while the first diffracted orders of the main beam are just replicas of the zeroth order in the case of a plain sinusoidal plasma grating (Fig. 2b), their intensity profiles get an 

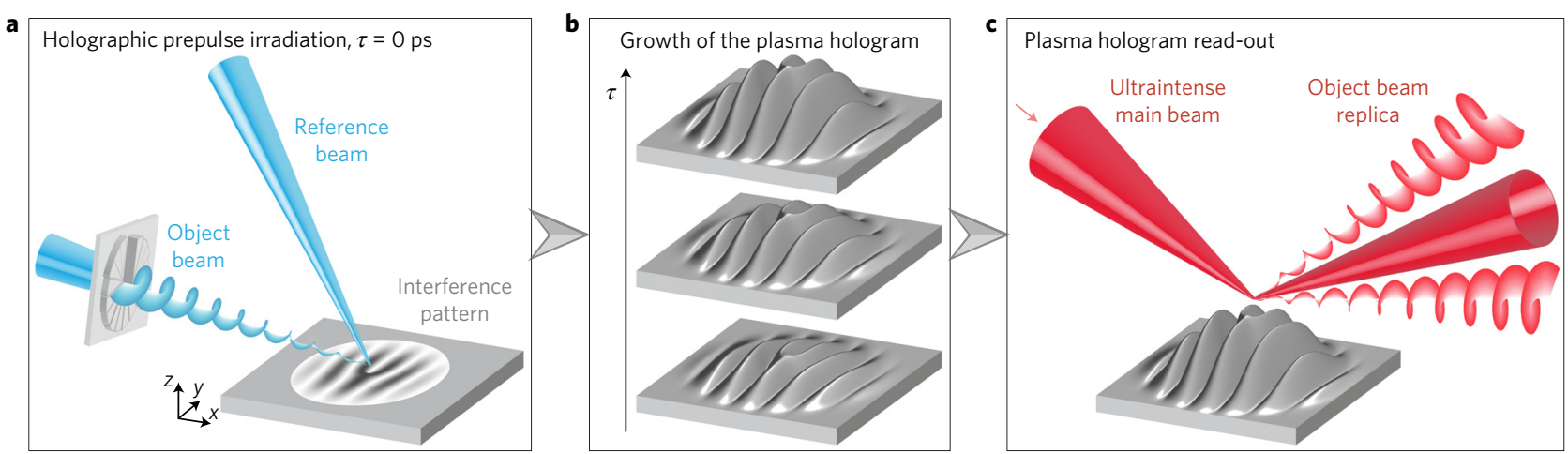

Figure 1 | Principle of plasma holograms. a, An initially flat solid target is ionized by an intense holographic pattern (peak fluence of $\approx 10^{3} \mathrm{~J} \mathrm{~cm}^{-2}$ in bright fringes), obtained by crossing a reference and an object beam (in blue), both consisting of femtosecond pulses. In this example, the holographic pattern is a fork grating (white and grey image in the target plane), produced by placing a spiral phase plate in the object beam. $\mathbf{b}$, The spatial modulation of the ionizing beam fluence leads to a modulation of the plasma expansion velocity. This results in the growth of a structured plasma surface in the picoseconds following ionization-in the present example, a plasma fork grating. c, This structured plasma surface can diffract an ultraintense femtosecond laser beam, leading to high-energy replicas of the initial object beam in the diffracted orders.
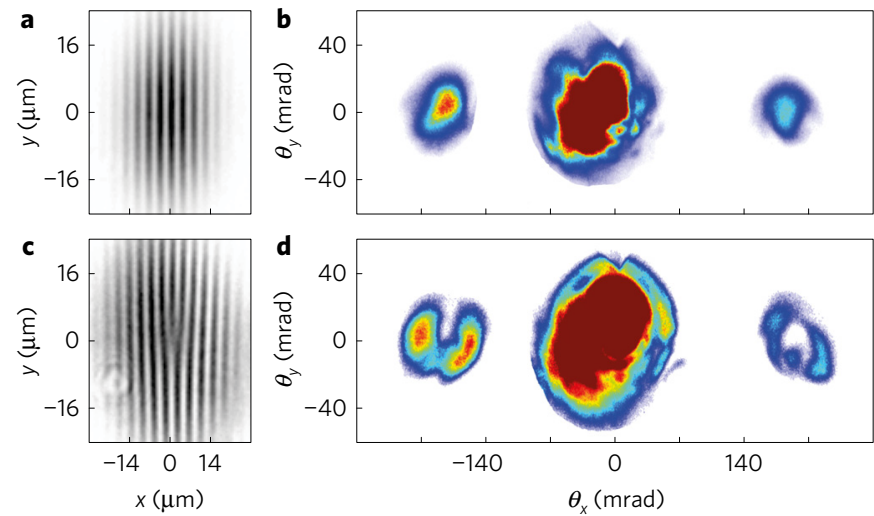

Figure 2 | Diffraction of an intense laser beam on plasma holograms. $\mathbf{a}, \mathbf{b}$, Case of a plain sinusoidal grating, created by the interference pattern of two identical beams (a), that leads to the diffraction pattern of $\mathbf{b}$ for the main beam. $\mathbf{c}, \mathbf{d}$, Case of a plasma hologram whose growth is initiated by a fork interference pattern $(\mathbf{c})$. The resulting plasma structure leads to the diffraction pattern of $\mathbf{d}$ for the main laser beam, where intensity profiles typical of optical vortices are observed on the first diffracted orders. The peak intensity of the main laser beam on the plasma hologram is $\approx 10^{17} \mathrm{~W} \mathrm{~cm}^{-2}$.

annular structure when the spiral phase plate is inserted into one prepulse beam (Fig. 2d). This is a clear indication that a fork plasma grating has indeed been created.

The use of high-power laser beams carrying an optical vortex to drive ultrahigh-intensity laser-plasma interactions has recently attracted a strong interest from the theoretical point of view ${ }^{18-22}$. Yet, inducing optical vortices on such lasers gets increasingly challenging as their peak power increases ${ }^{23,24}$, as these large, powerful and broadband beams cannot be handled by conventional beam shaping techniques. The results of Fig. 2 demonstrate that this can be achieved by diffraction off fork plasma gratings, even at extremely high peak intensities. Yet, in the demonstration experiment of Fig. 2, the diffraction efficiency is weak $(\approx 6 \%)$, which could be a drawback of this scheme. However, this efficiency can be strongly increased by using larger grating modulation depths $\Delta x$. This is achieved experimentally by increasing the delay $\tau$ between the prepulses and the main pulse, since $\Delta x=\Delta v \tau$, with $\Delta v$ being the difference in the expansion velocity of the critical density surface between bright and dark prepulse fringes.
This is illustrated in Fig. 3, which shows the diffraction pattern produced when the laser beam diffracts off a simple plasma grating, as a function of $\tau$. As expected theoretically for a sinusoidal phase grating ${ }^{25}$, the intensity of the $k$ th diffraction order varies as $\left|J_{k}(\Delta \Phi)\right|^{2}$, where $\Delta \Phi=4 \pi \cos \theta \Delta x / \lambda_{\mathrm{L}}$ (with $\theta$ being the angle of incidence, and $\lambda_{\mathrm{L}}$ being the laser wavelength) is the amplitude of the spatial phase modulation induced by the grating on the reflected laser beam, and $J_{k}$ is the Bessel function of order $k$. Consequently, when $\Delta \Phi \approx 2 \mathrm{rad}$, the energy in order 1 (or -1 ) is about $40 \%$ of that of the beam reflected by a plain plasma mirror, while the zeroth order almost cancels. The diffraction efficiency in a single order could in principle even approach the reflectivity of a plain plasma mirror (from $70 \%$ (ref. 6) up to $96 \%$ (ref. 26)) by creating blazed plasma gratings, whose expansion could be triggered by the sawtooth-shape light interference pattern resulting from the crossing of $N>2$ adequately phased prepulse beams.

Until now, we have considered plasma holograms as linear optical elements. At intensities higher than $\approx 10^{16} \mathrm{~W} \mathrm{~cm}^{-2}$, the response of dense plasma surfaces to the laser field is known to become strongly nonlinear, leading to the generation of high-order harmonics of the laser beam ${ }^{10,11}$. These harmonics can be generated on plasma gratings as well, leading to a diffraction pattern on the harmonic beam $^{13}$. We now demonstrate that, like in the case of the laser beam, fork plasma gratings can also be exploited to induce optical vortices on the diffracted harmonic beams, thus providing extreme ultraviolet (XUV) vortices that are challenging to obtain by more conventional means.

To perform this experiment at the highest possible intensity, we now focus almost the entire main laser beam on the plasma hologram, reaching peak intensities estimated to $3 \times 10^{19} \mathrm{~W} \mathrm{~cm}^{-2}$. High-order harmonics are then generated by the relativistic oscillating mirror mechanism, that is, by the periodic Doppler upshift induced on the reflected laser waveform by the laser-driven relativistic oscillation of the plasma mirror surface $e^{27-29}$. The main beam focal spot now has a diameter of $5 \mu \mathrm{m}$, and covers less than two lines of the $d=3.75 \mu \mathrm{m}$ plasma grating. In this regime, the angular dispersion of the grating is too weak to separate adjacent diffracted orders, neither for the fundamental laser frequency nor for its harmonics, so that these orders spatially overlap and interfere (see Supplementary Information), as in the experiments of refs 13,15 . This peculiar diffraction regime makes it difficult to unambiguously diagnose optical vortices induced by a fork grating on the diffracted high-order harmonics.

We circumvent this issue by decreasing the line spacing of the grating, to increase its angular dispersion. To this end, we 

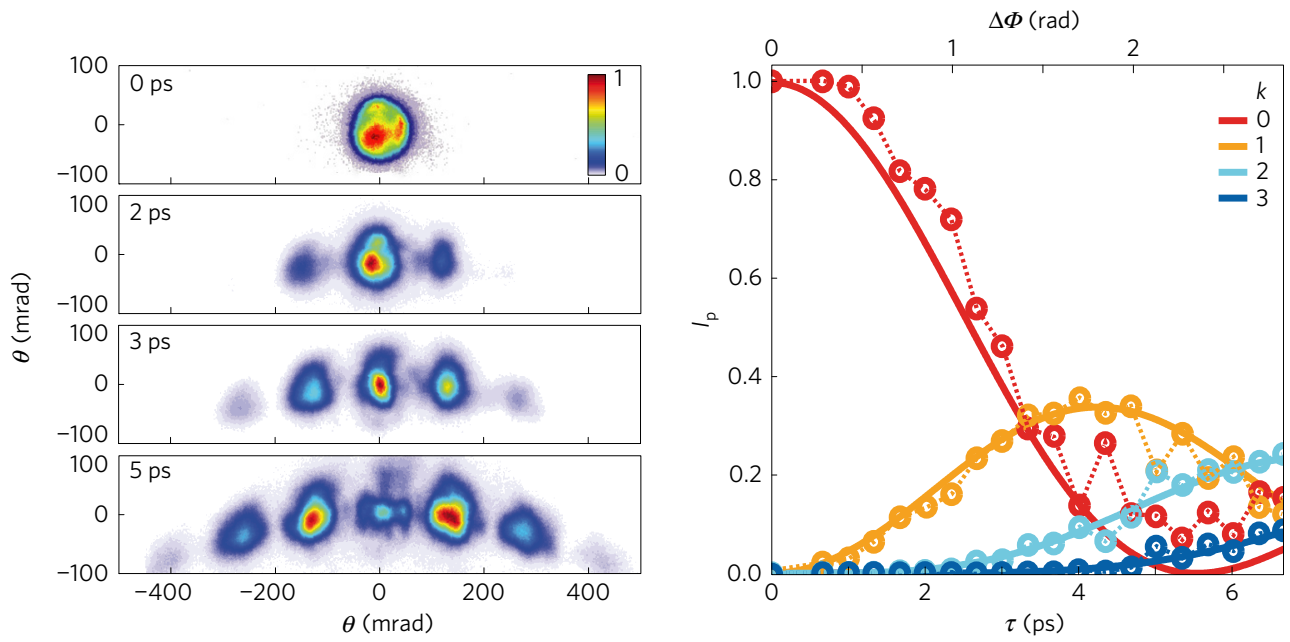

Figure 3 | Diffraction of an intense laser beam on plasma gratings of different depths. Left: laser diffraction patterns for different prepulse delays $\tau$, corresponding to different modulation depths $\Delta x=\Delta v \tau$ of the plasma grating (with $\Delta v=48 \mathrm{~nm} \mathrm{ps}{ }^{-1}$ ). These measurements have been performed in the conical diffraction geometry, thus leading to the observed curvature of the diffraction pattern. Right: diffracted energy in orders 0 to 3 , as a function of delay $\tau$ (lower axis), or of $\Delta \Phi=4 \pi \cos \theta \tau / \lambda_{\mathrm{L}}$, the amplitude of the sinusoidal spatial phase modulation induced by the grating on the laser beam (upper axis). The dotted lines and points shows the experimental results, and the full lines the fit of these data by $\left|J_{k}(\Delta \Phi)\right|^{2}$, with $k$ being the diffraction order and $J_{k}$ being the Bessel function of order $k$. The vertical axis has been normalized to 1 for the beam reflected by a plain plasma mirror (that is, order 0 at $\tau=0$ ).

frequency-double the prepulse beams using the compact and simple optical assembly described in the Supplementary Information. This divides the grating line spacing by a factor of two. The typical frequency-resolved diffraction pattern of the harmonic beam obtained with such gratings of spatial period $d / 2$ is shown in Fig. 4a, in the case of a plain sinusoidal grating. Adjacent diffracted orders are spatially well-separated.

Next, we create an $l=2$ fork plasma grating of line spacing $d / 2$, by placing the same helical waveplate as before in one of the frequency-doubled beamlets. The resulting harmonic diffraction pattern is displayed in Fig. $4 \mathrm{~b}$ : a dip is observed in the centre of the first diffracted orders, which is not present on the zeroth order, nor in the case of the plain sinusoidal grating. Like in the case of the laser beam, this is a clear indication that an optical vortex has been induced on the diffracted harmonic beamlets. The rather low modulation depth of this dip is due to the fact that the width of the entrance slit of the harmonic spectrometer $(1 \mathrm{~mm})$ was not negligible compared with the size of the ring-shape harmonic beam on this slit $(5 \mathrm{~mm})$. We note that in this case the plasma hologram modulates not only the spatial phase profile of the harmonic source, but also its spatial amplitude profile. Indeed, the density gradient scale length at the plasma-vacuum interface varies spatially, leading to modulations of the harmonic generation efficiency across the hologram. Numerical simulations based on a simple model indicate that this does not affect at all the quality of the produced harmonic vortices, whatever the relative weight of these two modulations (see Supplementary Information). In this holographic scheme, the key factor is the fork spatial structure of the hologram, be it in phase or in amplitude.

Visible or near-visible Laguerre-Gaussian beams have found groundbreaking applications in different fields in the last 15 years, due both to their doughnut-shape intensity profile ${ }^{30}$, and to the fact that they carry an angular orbital momentum of $l \hbar$ per photon ${ }^{17}$. More recently, many efforts have been devoted to the generation of such vortices at much shorter light wavelength ${ }^{31-34}$, which might also find far-reaching applications but are technically challenging to produce. To this end, a rather straightforward approach consists of generating high-order harmonics of a low- $l$ Laguerre-Gaussian laser beam, using gases ${ }^{31-33}$ or plasmas ${ }^{21}$ as a nonlinear medium. Due to the conservation of angular orbital momentum, this generates
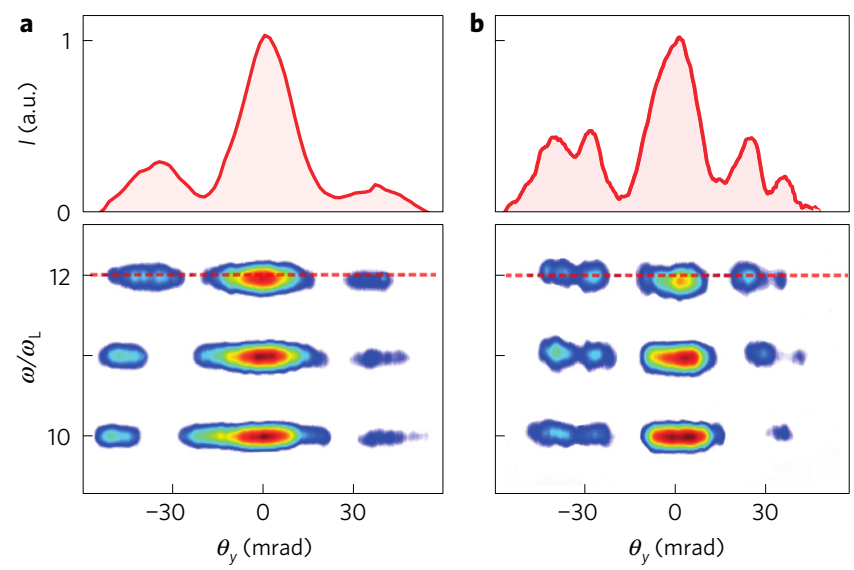

Figure 4 | Generation of high-order harmonic beams on plasma holograms. a, Case of a plain sinusoidal grating of line spacing $d / 2$, created by the interference of two frequency-doubled prepulses. The angular dispersion of this grating is sufficient to angularly separate adjacent diffracted orders for all harmonics. b, Case of a fork grating, created by inserting a spiral phase plate in one of the frequency-doubled prepulses. The dip observed in the first diffracted orders is a signature of the optical vortices of charge $I=2$ induced on these harmonic beamlets.

XUV Laguerre-Gaussian beams with a topological charge that is $n \times l$ for the $n$th harmonic beam ${ }^{32,33}$. This scheme thus produces XUV beams with high $l$ values, which increase with harmonic order and cannot be easily controlled.

The present approach provides a simple alternative approach to generate intense XUV vortex beams, with the key advantage that these have constant and adjustable $l$, which can be as low as 1 . Combined with even smaller grating line spacing, this can produce Laguerre-Gaussian beams made of a single harmonic, without the need for any additional optical element for spectral selection after the laser-plasma interaction. Conversely, if additional optics can subsequently be used to compensate the angular dispersion induced by the fork grating, attosecond pulses with an optical vortex of constant topological charge over their entire spectral width could be obtained. 
In conclusion, the present work establishes transient plasma holograms as a new category of plasma-based optical elements suitable for the efficient manipulation of ultraintense laser beams, both in the linear and nonlinear response regimes. We have exploited this approach to induce optical vortices on high-power femtosecond pulses and their high-order harmonics. Among other possible applications, one might consider using optimized (that is, blazed) plasma gratings as high-dynamic temporal filters to considerably improve the temporal contrast of high-power femtosecond lasers, and/or to carry out the final temporal compression in chirpedpulse-amplification lasers from the picosecond to the femtosecond range, thus relaxing the constraints imposed by the optical damage of conventional gratings. Simultaneously introducing spatial chirp on the prepulse beams at focus would produce varied-line-spacing plasma gratings, which could be exploited to achieve tight focusing of ultraintense beams.

Data availability. The data that support the plots within this paper and other findings of this study are available from the corresponding author on request.

Received 11 July 2016; accepted 7 December 2016; published online 16 January 2017

\section{References}

1. Thaury, C. et al. Plasma mirrors for ultrahigh-intensity optics. Nat. Phys. 3, 424-429 (2007).

2. Ren, J., Cheng, W., Li, S. \& Suckewer, S. A new method for generating ultraintense and ultrashort laser pulses. Nat. Phys. 3, 732-736 (2007).

3. Trines, R. M. G. M. et al. Simulations of efficient Raman amplification into the multipetawatt regime. Nat. Phys. 7, 87-92 (2011).

4. Turnbull, D. et al. High power dynamic polarization control using plasma photonics. Phys. Rev. Lett. 116, 205001 (2016).

5. Kapteyn, H. C., Murnane, M. M., Szoke, A. \& Falcone, R. W. Prepulse energy suppression for high-energy ultrashort pulses using self-induced plasma shuttering. Opt. Lett. 16, 490-492 (1991).

6. Doumy, G. et al. Complete characterization of a plasma mirror for the production of high-contrast ultraintense laser pulses. Phys. Rev. E 69, 026402 (2004).

7. Lévy, A. et al. Double plasma mirror for ultrahigh temporal contrast ultraintense laser pulses. Opt. Lett. 32, 310-312 (2007).

8. Hörlein, R. et al. High contrast plasma mirror: spatial filtering and second harmonic generation at $1019 \mathrm{~W} \mathrm{~cm}^{-2}$. New J. Phys. 10, 083002 (2008).

9. Nakatsutsumi, M. et al. Fast focusing of short-pulse lasers by innovative plasma optics toward extreme intensity. Opt. Lett. 35, 2314-2316 (2010).

10. Teubner, U. \& Gibbon, P. High-order harmonics from laser-irradiated plasma surfaces. Rev. Mod. Phys. 81, 445-479 (2009).

11. Thaury, C. \& Quéré, F. High-order harmonic and attosecond pulse generation on plasma mirrors: basic mechanisms. J. Phys. B 43, 213001 (2010).

12. Wheeler, J. et al. Attosecond lighthouses from plasma mirrors. Nat. Photon. 6, 829-833 (2012).

13. Monchocé, S. et al. Optically controlled solid-density transient plasma gratings. Phys. Rev. Lett. 112, 145008 (2014).

14. Kahaly, S. et al. Direct observation of density-gradient effects in harmonic generation from plasma mirrors. Phys. Rev. Lett. 110, 175001 (2013).

15. Leblanc, A., Monchocé, S., Bourassin-Bouchet, C., Kahaly, S. \& Quéré, F. Ptychographic measurements of ultrahigh-intensity laser-plasma interactions. Nat. Phys. 12, 301-305 (2016).

16. Bazhenov, V., Vasnetsov, M. \& Soskin, M. Laser beams with screw dislocations in their wavefronts. JETP Lett. 52, 1037-1039 (1990).
17. Yao, A. \& Padgett, M. Orbital angular momentum: origins, behavior and applications. Adv. Opt. Photon. 3, 161-204 (2011).

18. Vieira, J. \& Mendonça, J. T. Nonlinear laser driven donut wakefields for positron and electron acceleration. Phys. Rev. Lett. 112, 215001 (2014).

19. Zhang, G.-B. et al. Acceleration and evolution of a hollow electron beam in wakefields driven by a Laguerre-Gaussian laser pulse. Phys. Plasmas 23, 033114 (2016).

20. Wang, W. et al. Hollow screw-like drill in plasma using an intense Laguerre-Gaussian laser. Sci. Rep. 5, 8274 (2015).

21. Shi, Y. et al. Light fan driven by a relativistic laser pulse. Phys. Rev. Lett. 112, 235001 (2014).

22. Zhang, X. et al. Generation of intense high-order vortex harmonics. Phys. Rev. Lett. 114, 173901 (2015).

23. Brabetz, C. et al. Laser-driven ion acceleration with hollow laser beams. Phys. Plasmas 22, 013105 (2015).

24. Vieira, J. et al. Amplification and generation of ultra-intense twisted laser pulses via stimulated Raman scattering. Nat. Commun. 7, 10371 (2016).

25. Goodman, J. W. Introduction to Fourier Optics 3rd edn (Roberts and Company, 2005).

26. Scott, G. G. et al. Optimization of plasma mirror reflectivity and optical quality using double laser pulses. New J. Phys. 17, 033027 (2015).

27. Lichters, R., Meyer-ter-Vehn, J. \& Pukhov, A. Short-pulse laser harmonics from oscillating plasma surfaces driven at relativistic intensity. Phys. Plasmas 3, 3425-3437 (1996).

28. Baeva, T., Gordienko, S. \& Pukhov, A. Theory of high-order harmonic generation in relativistic laser interaction with overdense plasma. Phys. Rev. E 74, 046404 (2006).

29. Gonoskov, A. et al. Ultrarelativistic nanoplasmonics as a route towards extreme-intensity attosecond pulses. Phys. Rev. E 84, 046403 (2011).

30. Hell, S. W. \& Wichmann, J. Breaking the diffraction resolution limit by stimulated emission: stimulated-emission-depletion fluorescence microscopy. Opt. Lett. 19, 780-782 (1994).

31. Zürch, M., Kern, C., Hansinger, P., Dreischuh, A. \& Spielmann, C. Strong-field physics with singular light beams. Nat. Phys. 8, 743-746 (2012).

32. Gariepy, G. et al. Creating high-harmonic beams with controlled orbital angular momentum. Phys. Rev. Lett. 113, 153901 (2014).

33. Géneaux, R. et al. Synthesis and characterization of attosecond light vortices in the extreme ultraviolet. Nat. Commun. 7, 12583 (2016).

34. Hemsing, E., Marinelli, A. \& Rosenzweig, J. B. Generating optical orbital angular momentum in a high-gain free-electron laser at the first harmonic. Phys. Rev. Lett. 106, 164803 (2011).

\section{Acknowledgements}

The authors gratefully acknowledge F. Réau, C. Pothier and D. Garzella for operating the UHI100 laser, O. Gobert for his help in the alignment of the frequency-doubling system, and the mechanical workshop for its support. The research leading to these results has received funding from Agence Nationale pour la Recherche (project ANR-14-CE32-0011) and from the European Research Council under the European Union's Horizon 2020 research and innovation programme (ERC grant agreement 694596).

\section{Author contributions}

A.L., A.D., L.C. and F.Q. conceived the experimental set-up with the help of G.M., who contributed to the design of the frequency-doubling system. The experiment and the data analysis were carried out by A.L., A.D. and L.C. F.Q. proposed the initial idea, supervised the overall work with P.M., and wrote the paper with inputs from the other authors.

\section{Additional information}

Supplementary information is available in the online version of the paper. Reprints and permissions information is available online at www.nature.com/reprints.

Correspondence and requests for materials should be addressed to F.Q.

\section{Competing financial interests}

The authors declare no competing financial interests. 